\title{
Dosimetría absoluta mediante película radiocrómica en un sistema de irradiación gamma, utilizando un espectrofotómetro UV-VIS
}

\section{Absolute dosimetry through radiochromic film in gamma irradiation system using a spectrophotometer UV-VIS}

\section{Walter Vargas-Segura ${ }^{1}$}

Vargas-Segura, W. Dosimetría absoluta mediante película radiocrómica en un sistema de irradiación gamma, utilizando un espectrofotómetro UV-VIS. Tecnología en Marcha. Vol. 33-

1. Enero-Marzo. Pág 36-43.

doi) https://doi.org/10.18845/tm.v33i1.5019 


\title{
Palabras clave
}

Gafchromic HD-V2; calibración películas radiocrómicas; espectro absorbancia; irradiador gamma; densidad óptica.

\section{Resumen}

El propósito de este trabajo fue la calibración de las películas Gafchromic HD-V2 mediante la adaptación de los procedimientos de calibración de películas utilizados en dosis bajas. Las películas se irradiaron en 11 valores de dosis en el rango de 0 a 106,28 Gy en un sistema de irradiación gamma y utilizando un espectrofotómetro UV-VIS para medir la densidad óptica. Se obtuvieron los espectros de absorbancia para varias dosis y el pico de máxima absorbancia se ubicó en la longitud de onda de $677 \mathrm{~nm}$. Esta longitud de onda se utilizó para realizar las medidas de la densidad óptica (OD), y estos resultados se utilizaron para realizar el gráfico de dosis en función a la densidad óptica neta (ODn). Se obtuvieron la curva de calibración de las películas y la incertidumbre en la dosis, con valores inferiores al 4\% en dosis entre 10 a 20 Gy, e incertidumbres inferiores al 3\% para dosis superiores a 20 Gy hasta completar el rango de la curva. La calibración del sistema película radiocrómica-espectrofotómetro se obtuvo para el rango de dosis y el sistema de irradiación gamma empleado.

\section{Keywords}

Gafchromic HD-V2; radiochromic film calibration; absorbance spectrum; gamma irradiator; optical density.

\begin{abstract}
The propose of this work was the Gafchromic HD-V2 film calibration, though adapting film calibration procedures applied in low dose. The films were irradiated in 11 dose values within of the range from 0 to 106,28 Gy in a gamma irradiation system, and using a spectrophotometer for the optical density measure. The absorbance spectrum were obtained for several dose, and the maximum absorbance peak was located in the wavelength of $677 \mathrm{~nm}$. This wavelength was used for make the measurements of the optical density (OD), and these results were used for make the dose versus net optical density (ODn) graph. The calibration curve of the films and uncertainty in the dose were obtained, with values under 4\% in dose between 10 to 20 Gy, and uncertainties under 3\% for dose up from 20 Gy until complete the range of the curve. The calibration of the radiochromic film-spectrophotometer system was obtained for dose range and gamma irradiation system employed.
\end{abstract}

\section{Introducción}

Las películas radiocrómicas Gafchromic HD-V2 cuenta con una capa activa de $8 \mu \mathrm{m}$ de espesor que se encuentra sobre una capa de poliéster de $97 \mu \mathrm{m}$ de espesor [1]. La sensibilidad a la radiación de la capa activa depende de su espesor, que al ser expuesta se genera un proceso de polimerización, el cual produce un cambio en las propiedades ópticas de la película, es por esta razón que se establece una relación entre la densidad óptica (OD) de la película y la dosis recibida [2].

El rango de dosis para la película HD-V2 es de 10 a 1000 Gy, por lo que su uso no suele ser en el ámbito médico, sino más bien en el industrial o investigación a altas dosis [3]-[5]. Se 
han realizado muchos estudios de los instrumentos utilizados para la medida de la OD de las películas, incluso trabajos comparativos entre distintos dispositivos, pero, en la gran mayoría se hace referencia a las películas radiocrómicas utilizadas en el ámbito médico [6]-[9]. Además, se han publicado procedimientos para el proceso de calibración de la películas radiocrómicas [10], [11].

Uno de estos instrumentos utilizados para la medida de la OD es un espectrofotómetro, pero este equipo suele usarse para el análisis del espectro de absorbancia de la película y en pocas ocasiones para realizar dosimetría absoluta [5], [12]-[15].

El propósito de este trabajo es la calibración de las películas radiocrómicas HD-V2, utilizando un espectrofotómetro UV-VIS como instrumento para la medida de la OD, basándose en los estudios realizados en la calibración de películas radiocrómicas utilizadas en el ámbito médico.

\section{Materiales y métodos}

Las películas Gafchromic HD-V2 (lote\#: 11171601) utilizadas en este trabajo, vienen en láminas de 8"x10". Esta lámina fue cortada en piezas de 1,5 cm x 2,5 cm. Se agruparon 3 piezas por dosis de irradiación, para un total de 33 piezas utilizadas en este estudio. Para ser irradiadas, cada grupo de 3 piezas fueron colocadas en un maniquí de forma cilíndrica, fabricado de polimetilmetacrilato (PMMA) con dimensiones de $0,8 \mathrm{~cm}$ de radio interno, $1,2 \mathrm{~cm}$ de radio externo y $6,5 \mathrm{~cm}$ de altura.

Las películas fueron irradiadas en un sistema de irradiación gamma de la marca Izotop, modelo Servo-Ignis de fabricación húngara, que cuenta con 24 lápices de cobalto 60. La cámara de irradiación es de forma cilíndrica de dimensiones de $15 \mathrm{~cm}$ de diámetro, por $27 \mathrm{~cm}$ de alto, para una capacidad de 5 litros. Para el momento de este estudio, la tasa de dosis en el centro de la cámara era de $51 \mathrm{~Gy} / \mathrm{min}$. Se tomaron 11 de valores de dosis para irradiar las películas en el rango de 0 a 106,28 Gy, colocando el maniquí con las 3 películas en el centro de la cámara de irradiación.

El proceso de lectura de las películas radiocrómicas se realizó 24 horas después de haber sido irradiadas, este período de tiempo es necesario para que se estabilice el proceso de polimerización [11]. Para medir el grado de oscurecimiento de las películas debido a la dosis recibida, se utilizó un espectrofotómetro UV-VIS Evolution 220 marca Thermo (Thermo Fisher), en modo absorbancia. Primero, a las películas irradiadas a distintas dosis se les tomaron los espectros de absorbancia en la región visible del espectro electromagnético (400 $\mathrm{nm}$ a $750 \mathrm{~nm}$ ), con el fin de determinar la longitud de onda del pico de mayor absorbancia. Además, para observar posibles distorsiones en el espectro (ruido), así como la capacidad del espectrofotómetro para medir el grado de oscurecimiento de la película, dada por la limitación indicada por el fabricante de 3,0 en la medida de la absorbancia.

Hallada la longitud de onda del pico de mayor absorbancia, se realizaron las medidas de las películas tomando la densidad óptica igual a la absorbancia. En este procedimiento se realizaron 3 medidas por películas, manteniendo la misma orientación de las películas para evitar el efecto de la polarización [16], [17]. Por lo que, por dosis de irradiación, se tomó el valor promedio de las nueve medidas de OD realizadas, calculando además, la incertidumbre asociada a este valor promedio [18]. Pero, para la construcción de la curva de calibración, se calculó la densidad óptica neta (ODn), mediante la siguiente ecuación:

$$
O D n=O D_{i r}-O D_{\text {sin }}
$$

Donde:

$O D_{i r r^{\prime}}$ es la densidad óptica de la película irradiada. 
$O D_{\text {sin }}$, es la densidad óptica de la película sin irradiar (0 Gy).

Para la construcción de la curva de calibración de las películas, se graficó la dosis en función de la ODn. El ajuste propuesto para esta curva y la incertidumbre asociada a los valores de dosis que se deriven de esta calibración, se siguió con lo establecido por Devic et al [15]. El cual menciona en su trabajo, una ecuación para el ajuste de la curva, de la forma:

$$
D_{\text {Ajuste }}=a * O D n+b * O D n^{n}
$$

Donde:

ODn, es la densidad óptica neta.

$D_{\text {Ajuste }}$ es la dosis calculada a partir del valor de ODn medido.

a y b, son los parámetros del ajuste y n es un valor constate determinado por el usuario.

Además, en su trabajo se establece la expresión para la incertidumbre de la dosis, de la forma:

$$
\sigma_{D}(\%)=\frac{\sqrt{O D n^{2} \cdot \sigma_{a}^{2}+O D n^{2 n} \cdot \sigma_{b}^{2}+\left(a+n \cdot b \cdot O D n^{n-1}\right)^{2} \cdot \sigma_{O D n}^{2}}}{D_{\text {Aiuste }}} \cdot 100
$$

Donde:

$\sigma_{D}, \sigma_{O D n}, \sigma_{a}$ y $\sigma_{b}$ son las incertidumbres asociadas: a la dosis, a la ODn y a los parámetros del ajuste a y b, respectivamente.

$D_{\text {Ajuste }}$ es la dosis calculada mediante la curva de calibración.

Para el tratamiento de los datos y la elaboración de las gráficas se utilizaron los programas Excel y GNUPlot.

\section{Resultados}

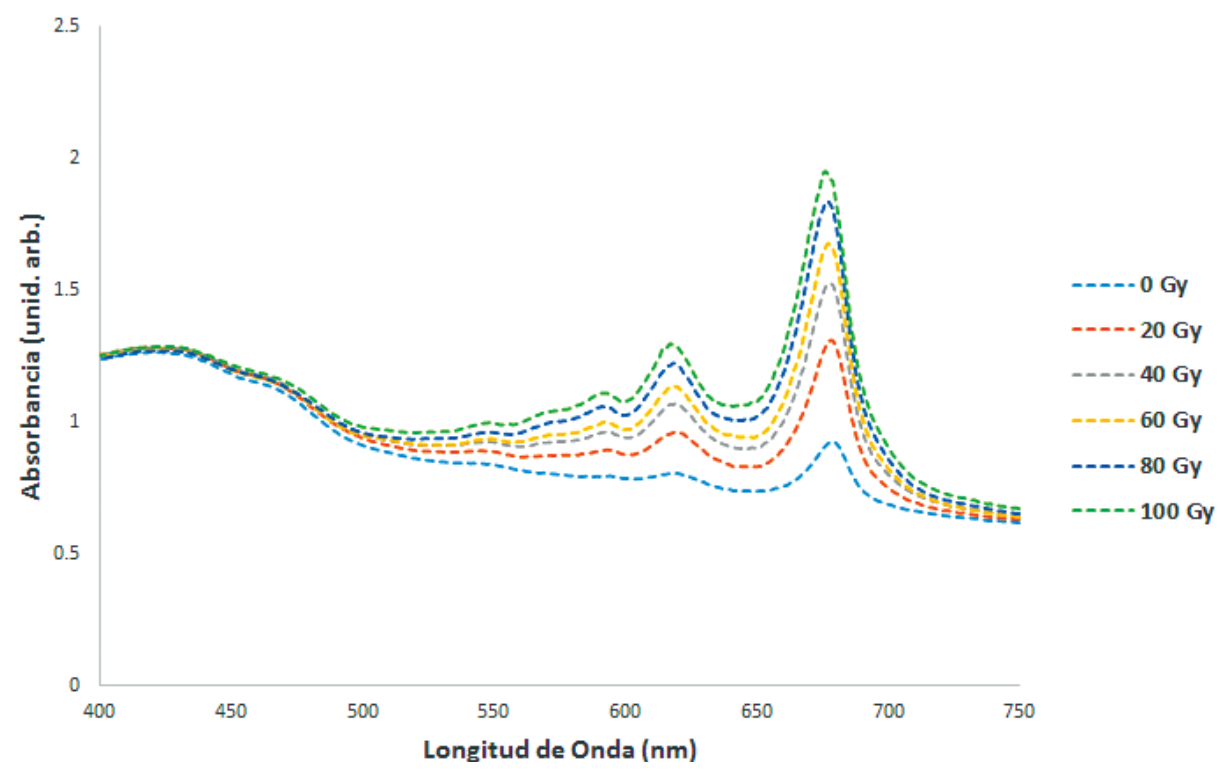

Figura 1. Espectros de absorbancia a distintas dosis. 


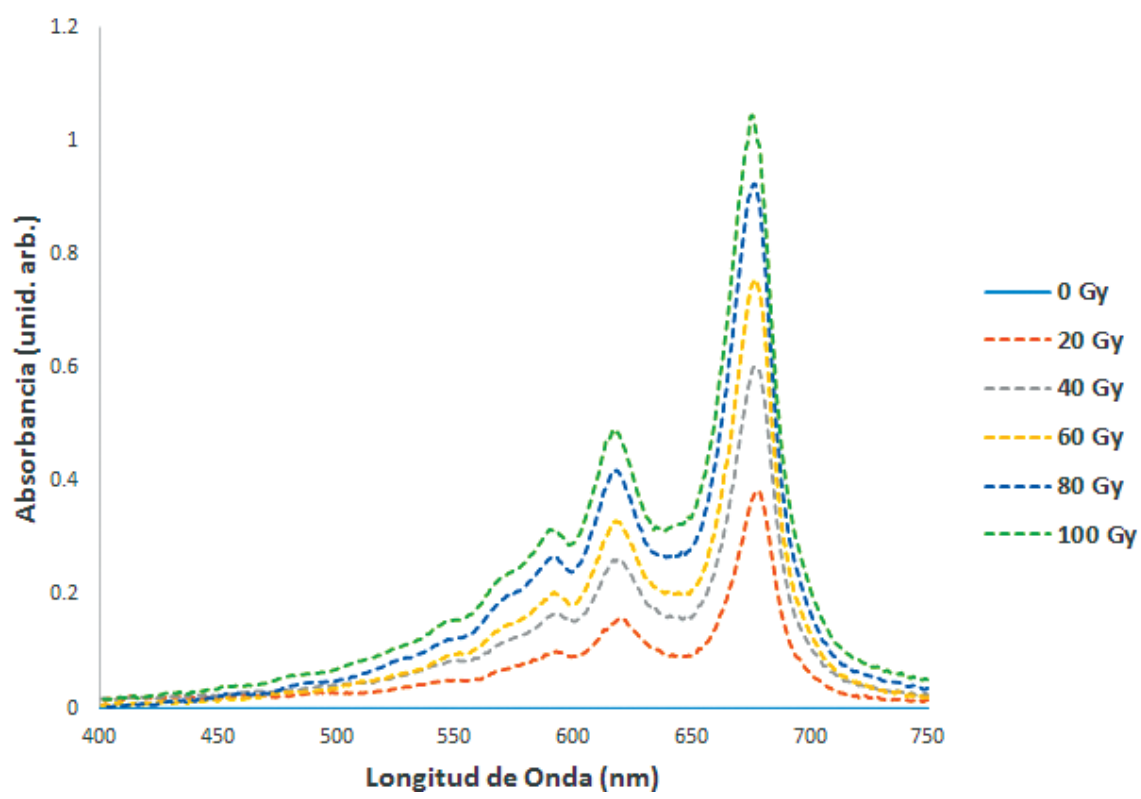

Figura 2. Espectros de absorbancia neta. Es el resultado de la diferencia entre la absorbancia a una dosis determinada menos la absorbancia de la película sin irradiar (0 Gy).

En la figura 1 se muestran los espectros de absorbancia a distintas dosis. Donde se puede localizar el pico de mayor absorbancia en la longitud de onda de $677 \mathrm{~nm}$. De esta figura se puede apreciar que la absorbancia aumenta con la dosis y también que no existen distorsiones en el espectro, ni cortes en los picos de absorbancia. La figura 2 muestra el espectro de absorbancia neta, que es el resultado de la resta del espectro de la película irradiada, menos el espectro de la película sin irradiar (0 Gy), procedimiento que fue utilizado para obtener la ODn.

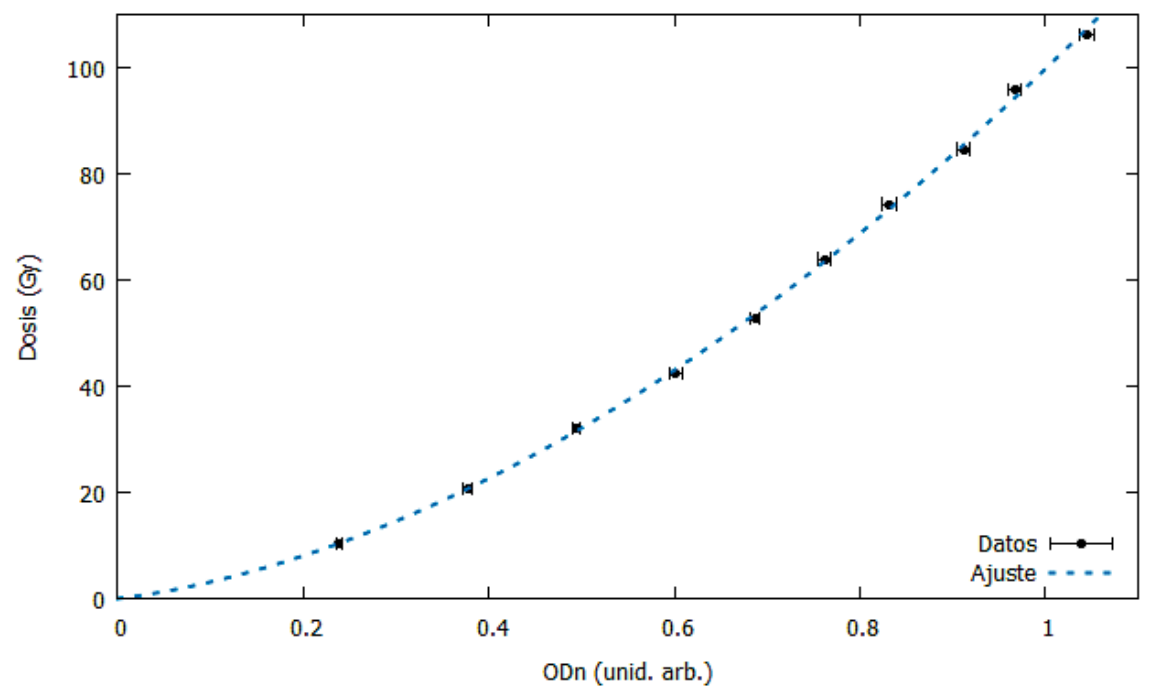

Figura 3. Curva de calibración de las películas HD-V2.

La figura 3 presenta la curva de calibración con el ajuste propuesto. Se obtuvo una correlación por encima del 0,999. 


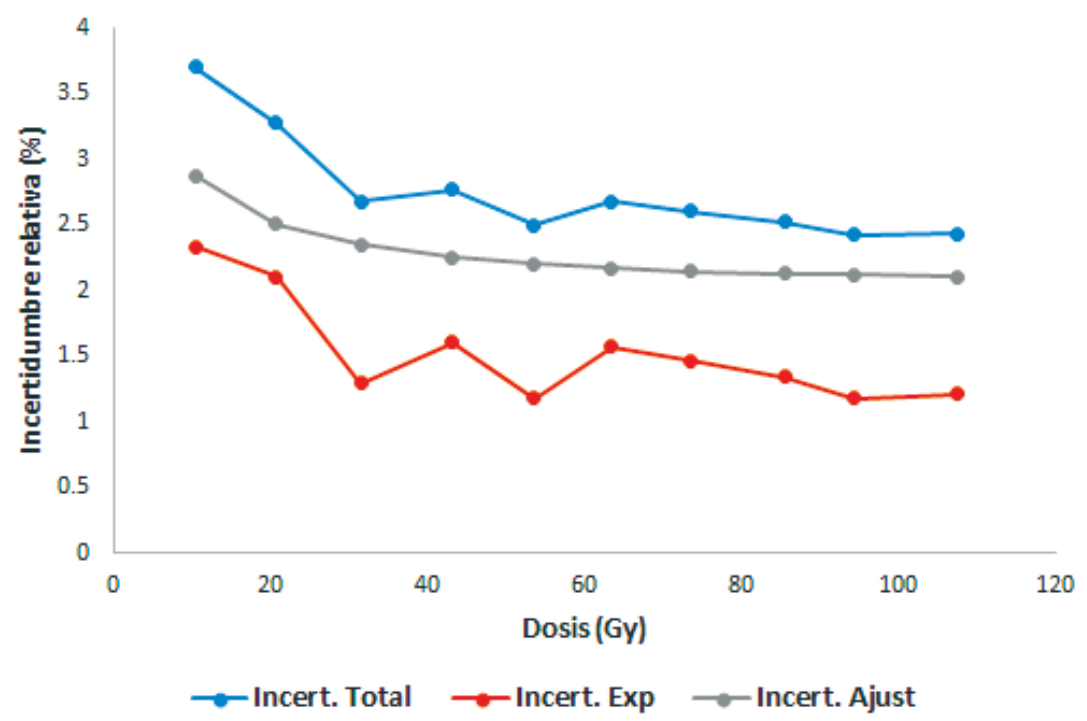

Figura 4. Variación de la incertidumbre en función de la dosis.

La variación de la incertidumbre en la dosis calculada a partir de la curva de calibración se muestra en la figura 4. La cual presenta una disminución de la incertidumbre al aumentar la dosis.

\section{Discusión}

En este estudio se toman procedimientos empleados en la calibración de películas radiocrómicas utilizadas a bajas dosis y son aplicados en el proceso de calibración espectrofotómetro-película radiocrómica $\mathrm{HD}-\mathrm{V} 2$, que serán utilizados como sistema de dosimetría de rutina a material irradiado en un sistema de irradiación gamma.

Los espectros presentados muestran el pico de mayor absorbancia localizado en la longitud de onda de $677 \mathrm{~nm}$ y un comportamiento creciente de la OD al aumentar la dosis, ya que es en esta región donde se presentan las mayores diferencias de OD entre dosis, lo cual reduce la posibilidad que exista superposición de espectros y el error en la determinación de la dosis, este resultado concuerda con otros estudios [3], [5]. Además, los valores máximos de OD no sobrepasaron la tolerancia del detector dada por el fabricante, lo que no generó distorsiones en los espectros de absorbancia medidos. El sobrepasar el límite genera un corte en el pico de absorbancia y un aumento en el ruido, lo anterior debido a la capacidad del detector, ya que la cantidad de luz que atraviesa la película y llega al detector es menor a su límite de detección [5], [13]. Yiwei Feng et al. [5] presenta esta limitación en su estudio del espectro de absorbancia para estas películas al ser irradiadas con protones a altas dosis y sugiere el uso de otras longitudes de onda para la determinación de la OD.

Hay varios estudios en que el uso del espectrofotómetro se limita al análisis del espectro absorbancia de las películas [12]-[15], y la dosimetría absoluta es realizada mediante otro tipo de equipos como los escáner de mesa [19], [20], tal es el caso de S. N. Chen et al [4], que en su estudio las películas radiocrómicas utilizadas (entre ellas la HD-V2), fueron medidas mediante diversos escáneres de mesa en modo RGB y en escala de grises, presentado las curvas de calibración para cada canal y propone ajustes polinomiales de segundo y tercer grado para las curvas. Respecto a la ecuación de ajuste, se observa en la literatura la aplicación de diversas funciones, quedando la escogencia a criterio del investigador [4], [9], [10], [15]. Para el caso 
de este trabajo, se inclinó por el ajuste planteado en la metodología debido a que los valores calculados de ODn permitieron construir la curva de calibración, obteniendo como resultado un ajuste aceptable, al igual que las incertidumbres obtenidas. Que relacionado a este último aspecto, no fue posible encontrar en la literatura un tratamiento detallado de la incertidumbre asociada a la dosis calculada mediante la curva de calibración, tanto para este tipo de películas [3], [4], como en estudios en que se utiliza un espectrofotómetro como instrumento de lectura de la OD [3], [5], [12]. Como se indicó anteriormente, esto motivó a que se utilizara el procedimiento planteado por Devic et al. [15] para el tratamiento de la incertidumbre asociada a la dosis y al tipo de ajuste propuesto, que al comparar el resultado obtenido con su estudio, se observa un comportamiento similar en la incertidumbre, que tiende a ser mayor a bajas dosis (cerca del límite inferior de detección de la película), y luego se reduce al aumentar la dosis. Este comportamiento también lo presenta E. Marroquin et al. [21] en su estudio acerca de la incertidumbre de las películas EBT3, que en el límite inferior de dosis, esta incertidumbre llega aproximadamente al $7 \%$ en el canal rojo, que es el utilizado para bajas dosis, hasta llegar a valores cercanos al $1 \%$ a dosis más altas. Un detalle importante es que el resultado obtenido en el límite inferior de detección para la película HD-V2 fue por debajo del 4\%, que es considerablemente menor al comparar con los estudios mencionados, además, el rango de dosis utilizado es un 10\% del rango total de detección de las películas HD-V2 (de 10 a 1000 Gy). Este resultado abre la interrogante para un posterior trabajo, que si lo anterior es debido al instrumento utilizado en la medida DO o es producto del comportamiento de la película, para ello se debe hacer un análisis comparativo entre un escáner de mesa de alta resolución y el espectrofotómetro.

La curva de calibración encontrada es válida para el sistema de dosimetría empleado (espectrofotómetro-película radiocrómica HD-V2) y para el irradiador gamma utilizado.

\section{Conclusión}

Por los resultados obtenidos se logra adaptar el proceso de calibración de las películas radiocrómicas utilizadas a bajas dosis, a las condiciones de irradiación y de lectura de las películas utilizadas en este estudio, lo que habilita al sistema espectrofotómetro-película radiocrómica HD-V2, para ser utilizado en el control dosimétrico de rutina del material irradiado en el sistema de irradiación gamma.

\section{Referencias}

[1] Ashland dosimetry systems. Gafchromic dosimetry media, type HD-V2, 2012.

[2] S. Devic et al. "Absorption spectroscopy of EBT model Gafchromic ${ }^{\text {TM }}$ film", Med. Phys., vol. 34, n 1, pp: 112118, 2007. DOI: 10.1118/1.2400615

[3] A. Aydarous et al. "The effects of electrons and photons irradiation on the optical and thermophysical properties of Gafchromic HD-V2 films", Results in Physics, vol. 6, pp: 952-956, 2016. http://dx.doi.org/10.1016/j. rinp.2016.11.025

[4] S. N. Chen et al. "Absolute dosimetric characterization of Gafchromic EBT3 and HDv2 films using commercial flat-bed scanners and evaluation of the scanner response function variability", Rev. Sci. Instrum., vol. 87, pp: 073301-1 a 073301-6, 2016. http://dx.doi.org/10.1063/1.4954921

[5] Y. Feng et al. "Spectral calibration of EBT3 and HD-V2 radiochromic film response at high dose using $20 \mathrm{MeV}$ proton beams", Rev. Sci. Instrum., vol. 89, pp: 043511-1 a 043511-12, 2018. https://doi.org/10.1063/1.4996022

[6] J. E. Matney et al. "Evaluation of a commercial flatbed document scanner and radiographic film scanner for radiochromic EBT film dosimetry”, Journal of Applied Clinical Medical Physics, vol. 11, n² 2, pp: 198-208, 2010.

[7] S. Devic et al. "Dosimetric properties of improved Gafchromic films for seven different digitizers", Med. Phys, vol. 31, n 9, pp: 2392-2401, 2004. DOl: 10.1118/1.1776691 
[8] H. Alnawaf et al. "Comparison of Epson scanner quality for radiochromic film evaluation", Journal of Applied Clinical Medical Physics, vol. 13, n 5, pp: 314-321, 2012.

[9] G. R. Gluckman and L. E. Reinstein. "Comparison of three high-resolution digitizers for radiochromic film dosimetry", Med. Phys., vol. 29, nº 8, pp: 1839-1846, 2002. http://dx.doi.org/10.1118/1.1485056

[10] S. Devic et al. "Reference radiochromic film dosimetry: Review of technical aspects", Physica Medica, vol. 32 , pp: 541-556, 2016. http://dx.doi.org/10.1016/j.ejmp.2016.02.008

[11] A. Niroomand-Rad et al. "Radiochromic film dosimetry: Recommendation of AAPM Radiation Therapy Committee Task Group 55", Med. Phys., vol. 25, n 11, pp: 2093-2115, 1998. http://dx. doi.org/10.1118/1.598407

[12] $\mathrm{H}$. Alnawaf et al. "Absorption spectra response of XRQA radiochromic film to x-ray radiation", Radiation Measurements, vol. 45, pp: 129-132, 2010.

[13] M. B. Callens et al. "Modeling the dose dependence of the vis-absorption spectrum of EBT3 Gafchromic ${ }^{\text {TM }}$ films", Med. Phys., vol. 44, nº 6, pp: 2532-2543, 2017. https://doi.org/10.1002/mp.12246

[14] E. Y. Marroquín et al. "Análisis de los espectros de absorción de las películas EBT2 y EBT3", Revista Mexicana de Ingeniería Biomédica, vol. 38, n 1, pp: 363-371, 2017. dx.doi.org/10.17488/RMIB.38.1.32

[15] S. Devic et al. "Precise radiochromic film dosimetry using a flat-bed document scanner", Med. Phys., vol. 32, n 7, pp: 2245-2253, 2005. DOI: 10.1118/1.1929253

[16] M. J. Butson et al. "Polarization effects on a high-sensitivity radiochromic film", Phys. Med. Biol., vol. 48, pp: N207-N211, 2003. stacks.iop.org/PMB/48/N207

[17] H. Alnawaf et al. "Scanning orientation and polarization effects for XRQA radiochromic film", Physica Medica, vol. 26, pp: 216-219, 2010. doi:10.1016/j.ejmp.2010.01.003

[18] JCGM. "Evaluación de datos de medición-Guía para la expresión de la incertidumbre de medida", $3^{a}$ edición en español, 2009.

[19] V. Casanova et al. "Dosimetric characterization and use of Gafchromic EBT3 film for IMRT dose verification", Journal of Applied Clinical Medical Physics, vol. 14, n² 2, pp: 158-171, 2013.

[20] J. Lárraga-Gutiérrez et al. "Evaluation of a LED-based flatbed document scanner for radiochromic film dosimetry in transmission mode", Physica Medica, vol. 47, pp: 86-91, 2018. https://doi.org/10.1016/j.ejmp.2018.02.010

[21] E. Y. Marroquín et al. "Evaluation of the uncertainty in an EBT3 film dosimetry system utilizing net optical density”, Journal of Applied Clinical Medical Physics, vol. 17, n 5, pp: 466-481, 2016. 